\title{
Primary Central Nervous System Lymphoma: A Diagnostic and Therapeutic Challenge-Case Report and Short Review
}

\author{
${ }^{1}$ UMF Carol Davila Bucharest, Romania \\ ${ }^{2}$ Department of Neurology, Fundeni Clinical Institute, Romania \\ ${ }^{3}$ Department of Hematology, Fundeni Clinical Institute, Romania \\ ${ }^{4}$ Department of Radiology Fundeni Clinical Institute, Romania
}

Adriana Octaviana Dulamea ${ }^{1,2^{*}}$, Alina Buture ${ }^{2}$, Sorina Badelita ${ }^{3}$ and Ioana Lupescu ${ }^{1,4}$

Submission: May 06, 2017; Published: July 13, 2017

*Corresponding author: Adriana Octaviana Dulamea, UMF Carol Davila Bucharest, Department of Neurology, Fundeni Clinical Institute, Romania, Tel: +40723258672; Fax: +403180420; Email: adrianadulamea@gmail.com

\begin{abstract}
Primary central nervous system lymphoma (PCNSL) is a rare CNS tumor characterized, in immune competent patients, by solitary, homogenous lesions, usually located into the cerebral hemispheres or basal ganglia. Authors report a case of PCNSL with unusual magnetic resonance imaging (MRI) findings, resembling the lesions of acute disseminate encephalomyelitis. New advanced MR imaging techniques such as diffusion, perfusion, MRS may help to differentiate PCNSL from other CNS lesions, for the prognostic, and for treatment response monitoring. PCNSL treatment involves high dose chemotherapy including methotrexate and cytarabine combined with anti-CD20 antibodybased immunotherapy and whole brain radiotherapy.
\end{abstract}

Keywords: Primary central nervous system lymphoma; Magnetic resonance imaging; Non Hodgkin B-cell lymphoma; Diffusion weighted images (DWI)

Abbreviations: PCNSL: Primary Central Nervous System Lymphoma; NHLS: Non-Hodgkin Lymphomas; DLBCL: Diffuse Large B Cell Lymphomas; GCB: Germinal Center B-Cell-Like; ABC: Activated B-Cell-Like; MRI: Magnetic Resonance Imaging, DWI: Diffusion Weighted Images; MRS: Magnetic Resonance Spectroscopy; CT: Computed Tomography; ADEM: Acute Disseminated Encephalomyelitis

\section{Introduction}

In the WHO Classification of Tumours of Hematopoietic and Lyphoid Tissue the term "primary central nervous system lymphoma" (PCNSL) is now restricted to primary diffuse large B-cell lymphoma confined to the CNS (and/or to the eye) that occurs in immunocompetent patients. PCNSL represent approximately $2 \%$ of all primary CNS tumors and approximately $1 \%$ of all non-Hodgkin lymphomas (NHLs). 95\% of them are diffuse large B cell lymphomas (DLBCL) [1-4]. Based on immunohistochemical markers DLBCL are classified into two major subtypes: germinal center B-cell-like (GCB) and activated B-cell-like (ABC) [5]. GCB DLBCL might have a better prognosis than ABC DLBCL [6]. PCNSL are not associated with EpsteinBarr virus and affect older populations, with a median age of 55 years. Clinical presentation of PCNSL is characterized by focal neurological signs, neurocognitive dysfunction and impaired performance status. In immune competent patients lesions are usually solitary, located in a cerebral hemisphere, thalamus/basal ganglia, corpus callosum, periventricular region and cerebellum. Neuroimaging findings of CNS lymphoma can be atypical in patients who are immunodeficient or who have been treated with radiation, antineoplastic agents, or steroids [2]. Autopsy studies revealed that most PCNSL extensively infiltrate the brain [7-10]. Therefore PCNSL treatment includes whole brain radiotherapy and high-dose chemotherapy. Methotrexate combined with highdose cytarabine is currently regarded as standard treatment. Ongoing trials compare whole brain radiotherapy with high-dose chemotherapy followed by autologous stem cell transplantation as consolidation (NCT01011920, NCT00863460) [1,3,4]. AntiCD20 antibody-based immunotherapy as a component of highdose methotrexate-based induction programs may contribute to improved outcomes [11]. Prognosis is poor with a median survival of 17 to 45 months in immune competent patients, and only $20-30 \%$ of cases can be cured successfully [4,6]. 


\section{Open Access Journal of Neurology \& Neurosurgery}

\section{Case Report}

Authors report the case of a 61-years-old woman, without relevant family and medical history, presenting with vertigo and unsteady gait. Brain computed tomography and magnetic resonance imaging (MRI) were interpreted as normal and the patient improved with symptomatic treatment of vertigo. Two weeks later the patient presented progressive onset of behavioral changes, apathy, and unsteady gait. The neurological examination revealed: temporal and spatial disorientation, left sixth nerve palsy, left facial palsy, hypophonia, dysphagia, unsteady gait, left hemiparesis, right crural paresis, left limbs ataxia, lack of verbal and motor initiative, apathy, and thalamic aphasia. General examination was normal.

\section{Results}

The unenhanced brain CT scan showed hypoattenuating areas involving the genu of corpum callosum and the right lenticulo-capsular region (Figure 1). Brain MRI (Figure 2) revealed multiple lesions with hyperintense signal on T2/ FLAIR sequences, isointense on T1 sequences, moderate water restriction on diffusion weighted images (DWI), some of them with moderate contrast enhancement, imprecisely delimited, localized at the level of corpus callosum, bilateral front o-insular periventricular white matter, bilateral capsulo-lenticular, right caudat nucleus, and with nodular appearance in the anterior pole of frontal lobe, left midbrain, right pons, left cerebral peduncle. The infra and supratentorial location as well as the random involvement of deep and superficial structures of gray and white matter led to the differential diagnosis among acute disseminated encephalomyelitis (ADEM), lymphoma and gliomatosis cerebri. The involvement of corpus callosum and the periventricular lesions made the diagnosis of ADEM less probable. Typical MRI findings in brain lymphomas are: homogeneous, iso- to hypointense T1 wi mass; iso- to hyperintense but often hypointense to gray matter on $\mathrm{T} 2$ wi with vasogenic edema; contrast enhanced T1 wi: homogeneously enhancing lesion with involvement of corpus callosum; diffusion- DWI: restricted diffusion of lesions secondary to hypercellularity, some of these MRI aspects were encountered in the presented case. The glioma hypothesis was less plausible because glioma usually shows T1-hypointense mass within white matter, with central heterogeneous signal (necrosis, intratumoural hemorrhage), variable enhancement, typically peripheral and irregular, hyperintense on T2-sequences, surrounded by vasogenic oedema and including flow voids (Figure 3). The Blood biochemistry including lactate dehydrogenase, hemoglobin, hematocrit and cell blood count were within normal range. Cerebral spinal fluid analysis showed: 7 monocytes $/ \mathrm{mm}^{3}, 0.6 \mathrm{~g} / \mathrm{l}$ protein (normal range $=0.1-0.3 \mathrm{~g} / \mathrm{l}$ ) $48.8 \mathrm{mg} / \mathrm{dl}$ glucose (normal range $74-106 \mathrm{mg} / \mathrm{dl}$ ), negative bacteriological examination, negative 14-3-3 protein and absent oligoclonal bands. The bone marrow aspirate was normal, therefore a systemic lymphoma with secondary brain involvement was excluded. Enzyme- linked immunosorbent assay for detection of antibodies against human immunodeficiency virus (HIV), hepatitis B and $\mathrm{C}$ viruses, Epstein-Barr virus, herpes simplex virus 1 and 2, cytomegalovirus and JC virus revealed normal results. Immunoserology showed normal immunoglobulins (IgG, IgM, IgA, IgE). After corticosteroid treatment an important clinical remission occurred, but with moderate regression of lesions on brain MRI (Figure 4 \& 5). The anatomo-pathological examination of brain tissue obtained by brain stereotactic biopsy showed non Hodgkin lymphoma with large B cell. Immunohistochemical analysis revealed: lymphoma cells were positive for CD20 (diffusely distributed into the tumor) and negative for CD3, Ki67 was positive in more than $80 \%$ of tumor cells, and rare, reactive, small lymphocytes were present (Figure 3). Spinal cord MRI, contrast enhanced computed tomography of chest, abdomen and pelvis, and dilated eye examination were normal. The patient received four courses of chemotherapy combining high doses of cytarabine, methotrexate, idarubicin, dexamethasone, vincristine and ifosfamide followed by whole brain radiotherapy. The first course of chemotherapy produced an important clinical and neuroimaging remission but a new relapse occurred and the patient died 6 months later.

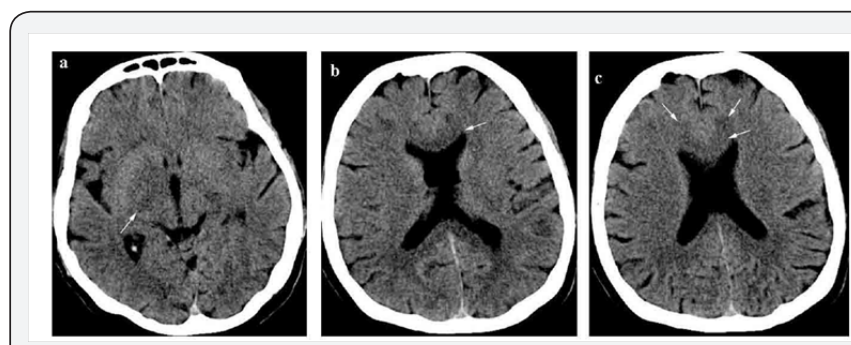

Figure1:(a,b,c)-UnenhancedCT(October15,2012): Hypoattenuating areas involving the genu of corpus collasum and the right lenticulo-capsular region.
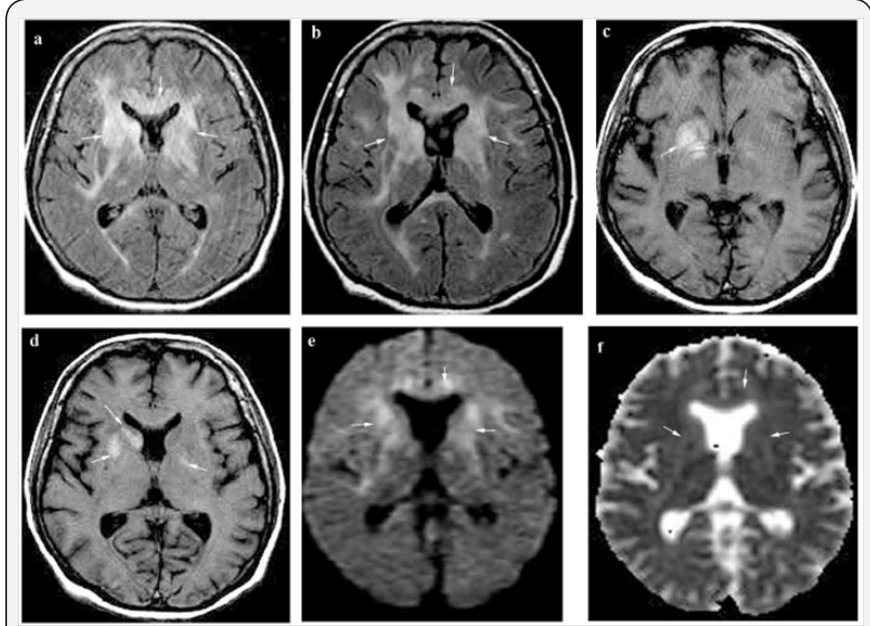

Figure 2 (a,b,c,d,e,f): MRI (October 19, 2012): Bilateral and asymmetric farce hyperintensities in Flair $(a, b)$ wi, diffusion (c) and ADC mop \{d\} involving the genu of corpus callosum, the white matter and the lenticulo-capsulo-caudate regions. NoteT1 $w$ hyperintense areas in the right lenticulo-caudate region and into the left globus pallidum $(e, f)$. 


\section{Open Access Journal of Neurology \& Neurosurgery}
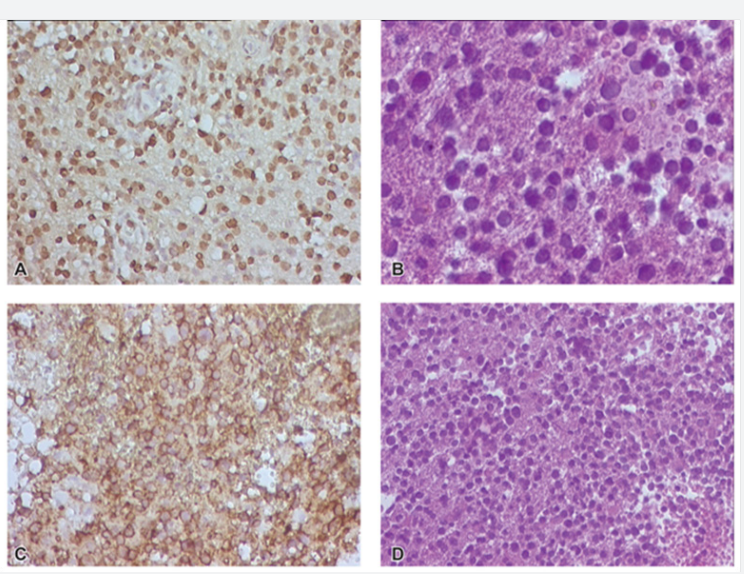

Figure 3: Immunohistochernical analysis of brain tissue: (A) High proliferation index Ki67 - 85-90\% (immunchistochernical stain for Ki67, ob 20x), (B) Lymphoid infiltrate with large, polymorph cell, with vesicular nucleus, visible nucleoli (Hematoxylin Eosin stain, ob 40x), (C) Tumor proliferation with B cell, diffuse positive for CD20 (immunohistochemical stain for CD20, ob20x), (D) Brain tissue with malignant lymphoid infiltrate with malignant lymphoid infiltarate with large cell(Hematoxylin Eosin stain, ob 20x).
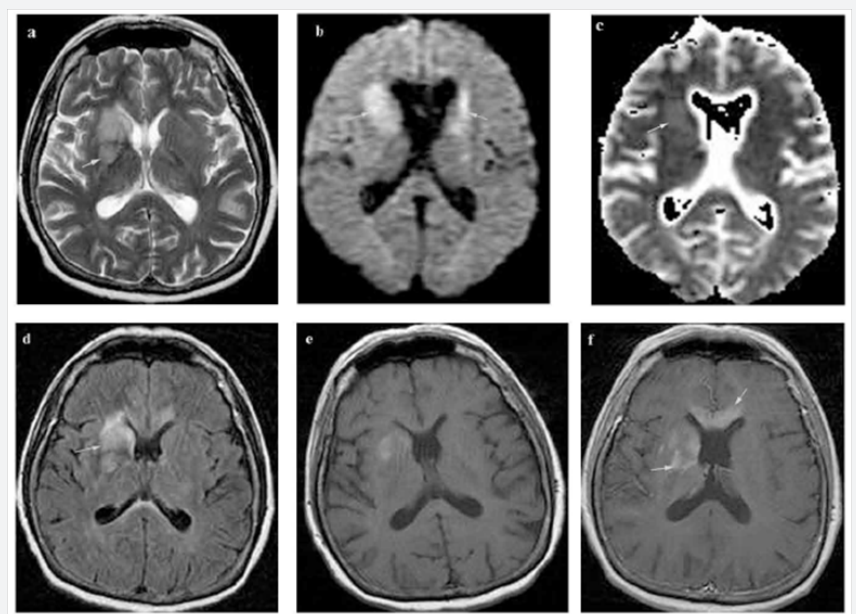

Figure 4 (a,b,c,d,e,f): MRI (November 28, 2012). Moderate regression of the hyperintensities areas in $\mathrm{T} 2 \mathrm{wi}(\mathrm{a})$, diffusion (b) ADC map (c) and Flair involving the genu of corpus callosum, and the lenticulo 7 caudate regions predominantly on the right side. Persistent T1 w hyperintense area in the right lenticulo $\urcorner$ caudate region (e), Contrast enhancement of the lesions involving the genu of corpus callosum and the right lenticulo-caudate region $(F)$.

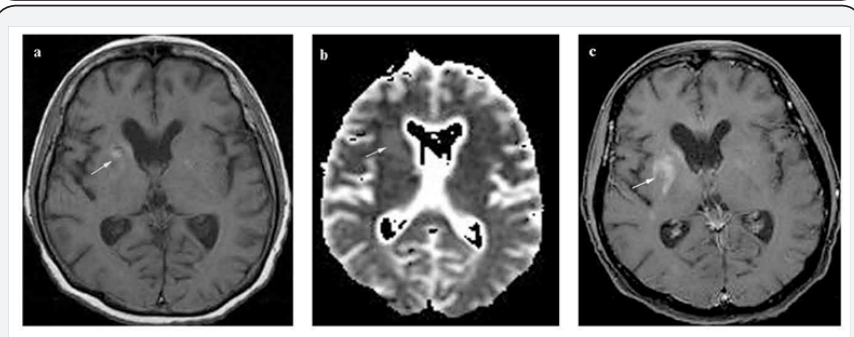

Figure 5 (a,b,c): MRI (January 28, 2013 ). Regression of lesions involving the genu of corpus callosum, and the lenticulo-caudate regions $(a, b)$, with persistent contrast enhancement in the right lenticular nucleus (c).

\section{Discussion}

The typical MRI lesions of PCNSL [8] are solitary, single or multiple, homogenous, with less prominent perilesional edema, located central hemispheric, in the periventricular white matter or superficial adjacent to the meninges, with intraocular involvement in $25 \%$ of cases. On precontrast $\mathrm{T} 1$ weighted images, lesions are usually isointense or hypointense with strong homogenous enhancement, on T2/FLAIR appear isointense, hyperintense or hypointense, on DWI are hyperintense and on apparent diffusion coefficient (ADC) are hypointense. Lesions can disappear after corticosteroids or even spontaneously, being called "vanishing tumors". Frontal lobe location is present in $20 \%-43 \%$ of PCNSLs, whereas the basal ganglia are involved in $13 \%-20 \%$. The corpus callosum is also frequently involved. In atypical imaging characteristics DWI, perfusion MRI and MR spectroscopy may help to differentiate CNS lymphomas from other brain lesions. DWI measures the diffusion of water molecules in biologic tissues and is considered a surrogate marker of tumor cellularity. Because PCNSL are highly cellular tumors, water diffusion is often restricted, making them appear hyperintense on DWI and hypointense on ADC maps. This characteristic is found also in: acute ischemic stroke, central necrosis of brain abscesses, the solid portion of high-grade gliomas, and some metastases. Nevertheless, PCNSL lesions have in most of cases more restricted diffusion and lower ADC values than high-grade gliomas and metastases [9]. Repeated ADC measurements may be used as biomarkers in the surveillance of therapeutic response of PCNSL [10]. Our case was particular because it showed that atypical, diffuse, imprecisely defined lesions, usually found in immunodeficient patients, especially HIV positive, can be also present in PCNSL in immunocompetent patients.

\section{Conclusion}

MRI is the best imaging method to evaluate and to follow-up PCNSL. Even though PCNSL may have typical imaging findings on classical MRI, none of them do not allow unequivocally to differentiate PCNSL from other brain lesions. New advanced MR imaging techniques such as diffusion, perfusion, magnetic resonance spectroscopy (MRS) may help to differentiate PCNSL from other CNS lesions, for the prognostic, and for treatment response monitoring. PCNSL treatment involves high dose chemotherapy including methotrexate and cytarabine combined with anti-CD20 antibody-based immunotherapy and whole brain radiotherapy.

\section{Acknowledgement}

Authors thank to Mrs. Melania Dulamea for assembling the images into figures.

\section{References}

1. Carnevale J, Rubenstein JL (2016) The Challenge of Primary Central Nervous System Lymphoma. Hematol Oncol Clin North Am 30(6): 1293-1316. 
2. Nabavizadeh SA, Vossough A, Hajmomenian M, Assadsangabi R, Mohan S (2016) Neuro imaging in Central Nervous System Lymphoma. Hematology/Oncology Clinics of North America 30(4): 799-821.

3. Laack NN, O’Neill BP, Ballman KV (2011) North Central Cancer Treatment Group and Mayo Clinic. CHOD/BVAM Chemotherapy and Whole-Brain Radiotherapy for Newly Diagnosed Primary Central Nervous System Lymphoma. International Journal of Radiation Oncology Biology Physics 81: 476-482.

4. Dolecek TA, Propp JM, Stroup NE, Kruchko C (2012) CBTRUS statistica report: Primary brain and central nervous system tumors diagnosed in the United States in 2005-2009. Neuro oncol 14(Suppl 5): 1-49.

5. Gerstner ER, Batchelor TT (2010) Primary central nervous system lymphoma. Arch Neurol 67: 291-297.

6. Bhagavathi S, Sharathkumar A, Hunter S, Sung L, Kanhere R, et al (2008) Activated B-cell immunophenotype might be associated with poor prognosis of primary central nervous system lymphomas. Clin Neuropathol 27(1): 13-20.
7. Lai R, Rosenblum MK, DeAngelis LM (2002) Primary CNS lymphoma: A whole-brain disease? Neurology 59(10): 1557-1562.

8. Haldorsen IS, Espeland A, Larsson EM (2011) Central nervous system lymphoma: characteristic findings on traditional and advanced imaging. AJNR Am J Neuroradiol 32(6): 984-92.

9. Toh CH, Castillo M, Wong AM, Wei KC, Wong HF, et al. (2008) Primary cerebral lymphoma and glioblastoma multiforme: differences in diffusion characteristics evaluated with diffusion tensor imaging. AJNR Am J Neuroradiol 29(3): 471-475.

10. Barajas RF, Rubenstein JL, Chang JS, Hwang J, Cha S (2010) Diffusionweighted MR imaging derived apparent diffusion coefficient is predictive of clinical outcome in primary central nervous system lymphoma. AJNR Am J Neuroradiol 31(1): 60-66.

11. Carnevale J, Rubenstein JL (2016) The Challenge of Primary Central Nervous System Lymphoma. Hematol Oncol Clin North Am 30(6): 1293-1316.

\section{Your next submission with Juniper Publishers} will reach you the below assets

- Quality Editorial service

- Swift Peer Review

- Reprints availability

- E-prints Service

- Manuscript Podcast for convenient understanding

- Global attainment for your research

- Manuscript accessibility in different formats

(Pdf, E-pub, Full Text, Audio)

- Unceasing customer service

Track the below URL for one-step submission https://juniperpublishers.com/online-submission.php 